\section{Remember then}

\author{
Peter Harman
}

Physicists Look Back. Studies in the His tory of Physics. Edited by John Roche. Hilger: 1990. Pp. 393. £45.

THE study of the history of physics has become a flourishing area of scholarship. As a manifestation of its wider impact, in recent years some physicists have expressed interest in the educational value of the subject. The present volume reflects this burgeoning interest within the physics community, and collects papers presented to meetings of the History of Physics group of the Institute of Physics. As a result, this is a very miscellaneous collection of pieces, and while some bristle with facts and bibliographic data (David Hughes on meteors), others have the charm of the after-dinner speech $(\mathrm{N}$. Kurti's reflections of an amateur historian). The editor has divided the papers as falling under three general themes.

The first part of the book is concerned with basic methods and includes papers on research libraries (Dennis F. Shaw), the techniques of oral history (D.H. DeVorkin), and the resources and archives of contemporary physics (Spencer Weart and John Krige). Ronald Stansfield's common-sense discussion of the real problems raised by claims to 'repeat' past experiments is enlivened by personal reminiscences of the laboratory culture of the 1930s.

The second theme ranges over the various issues raised by claims for the educational value of the history of physics. A. P. French gives a cautious appraisal of the role of history at university level, while Brian Davies and Stuart Leadstone outline several approaches to the incorporation of historical materials within a school syllabus. Whereas these papers offer personal views on the ways in which history can enrich physics teaching, John Roche claims that history has a leading role in clarifying the conceptual foundations of physics. In exemplification of this theme he gives an account of the history of the concept of the vector potential from its origins in nineteenth-century electromagnetic theory.

The third theme of the book includes reminiscences of personal contributions to contemporary physics. Pieces by E. Lipson (crystal structure), D. J. Unwin (electron probe instruments), Sir Granville Benyon (atmospheric research) and B. D. Josephson (superconductivity) are included here. Of these papers, B. D. Josephson's account of the work which led to the award of his Novel prize is likely to be of the greatest general interest. Josephson himself warns of the likely historical inaccuracy of his narrative, but such docu- ments can form a valuable part of the historical record.

The problem of confronting physics with its history is discussed by several contributors. A.P. French quotes Pasteur and Sir Peter Medawar in illustration of the fundamental problem. As Medawar put it, "Science therefore in some sense comprehends its history within itself". What then can be the role for history? In his Forces and Particles (Macmillan, 1972), Sir Brian Pippard concluded that "If we are to eschew a truly historical presentation, surely it is best to avoid the pretence of historicity altogether". It may well be that history of physics and physics no way pretends to be history as understood by historians, but draws upon their work to enrich the presentation. From the history side, Jed Buchwald's From Maxwell to Microphysics and The Rise of the Wave Theory of Light (Chicago University Press, 1985 and 1989 respectively), and Dan Siegel's Innovation in Maxwell's Electromagnetic Theory (forthcoming from Cambridge University Press), use the language of contemporary physics to describe and illuminate history, while remaining attentive to the integrity of the historical materials. This approach is not without its problems in characterizing historical nuances, but if the task of

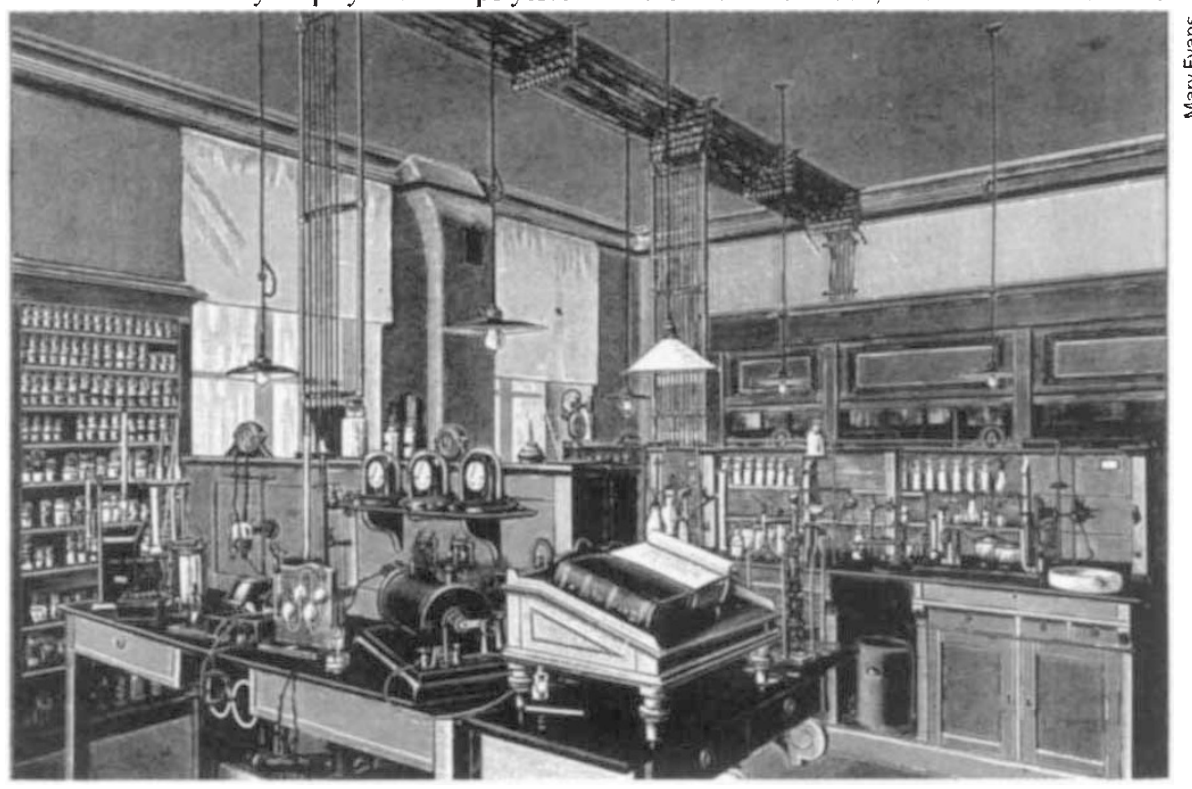

Laboratory culture - personal reminiscences enliven discussions of historical research.

are incommensurable. This would seem to be French's view, for he argues that "The presentation of history as historians understand it is close to being an impossibility in the physics class". Nevertheless, he believes that some historical materials can be used; these reduce to anecdotal vignettes, for we are engaged in 'the art of the possible"

John Roche makes claim for a radically different view of history as essential to physics itself. Yet in seeking to give an account of the vector potential in its historical development as a prelude to fundamental conceptual clarification, his account epitomizes the problem as stated by French. Roche's analysis conflates concepts and styles of mathematics which were historically distinct and had different meanings in their original context.

The problems raised by Medawar, Pippard and French need to be confronted by physicists who wish to use historical materials and by historians who wish to address their work directly to physicists. On the physics side, Malcolm Longair's Theoretical Concepts in Physics (Cambridge University Press, 1984) incorporates historical material into a sophisticated undergraduate text, in a style that in is to be undertaken, this work offers a solid foundation.

In his preface, Roche divides historical research into three categories: sources, social history and technical history. He considers that the present volume falls into the latter category, but I consider this a misleading designation. Physicists Look $B a c k$ does have its merits and interest, but it would be a pity if the physicists who are its likely readers came away with the impression that this is technical history as currently practised by historians of physics. Nor does the book give any indication of the scope of current work in the history of physics. To mention the study of sources alone, publication of the writings of the twentieth-century masters Bohr, Heisenberg, Pauli, and especially the Princeton Press edition of the Papers of Albert Einstein edited by Martin Klein and John Stachel, has been a major development during the $1980 \mathrm{~s}$. The editions of the works of the Bernoulli family and of Euler have recently taken on a new lease of life. Cambridge University Press are publishing Alan Shapiro's edition of Newton's optical papers, and D.T. Whiteside's recent facsimile volume of the Principia reappraising conceptual foundations 
manuscripts supplements his famous edition of the Mathematical Papers. Cambridge University Press has just published the first volume of my edition of The Scientific Letters and Papers of James Clerk Maxwell.

On a rather broader front, the literature abounds with interpretative studies, of which C. Jungnickel and R. McCormmach's study of German physics, Intellectual Mastery of Nature (Chicago University Press, 1986), stands pre-eminent among recent publications. It would be a pity if physicists wanting to make use of historical work remained unaware of all this effort.

P. M. Harman is a visiting scholar in the Department of History of Science, Harvard University, Cambridge, Massachussetts 02138, USA.

\section{Holy grail or snark?}

\section{J. Ellis}

The Higgs Hunter's Guide: Frontiers in Physics. By J. F. Gunion, H. E. Haber, G. Kane \& S. Dawson. Addison-Wesley: 1990. Pp.425. £41.35, \$49.50.

WHAT is the most pressing problem in particle physics today? All confirmed experimental results agree dramatically with the Standard Model, giving us no clue how to progress beyond it. But the Standard Model is very cumbersome and inelegant, containing three distinct sets of gauge interactions, nine a priori arbitrary quark and lepton masses, four assorted mixing angles and phases to describe the charged-current weak interactions, and two other parameters thrown in by hand to break spontaneously the electroweak symmetry group. The latter 15 parameters all describe the couplings and mass of the mythical Higgs boson, supposed purveyor of masses to all the other elementary particles, but as yet únseen by experiment and the subject of furious theoretical debate. These seem reasons enough to cite the quest for the Higgs boson as the most pressing problem in particle physics, though some might cite the unification of all the interactions and understanding the proliferation of different particle types (the "flavour problem"). However, I consider the origin of mass and the mechanism of symmetry breaking prerequisites for addressing these important problems, particularly because the reasons for thinking that the Higgs boson should be accessible to present or planned accelerators are better than those for expecting detectable manifestations of unification or flavour physics.

With the recent start-up of the Large
Electron-Positron Collider (LEP) and advanced plans for the Large Hadron Collider (LHC) accelerator in Europe and the Superconducting Super Collider (SSC) in the United States, this book reviewing strategies for detecting the Higgs boson is therefore particularly timely. Moreover, the authors are among the best qualified to write on this important topic. Indeed, they provide the reader with a large amount of useful and accurate information about the expected properties of the Higgs boson and how to search for it in $\mathrm{e}^{+} \mathrm{e}^{-}$and hadron-hadron collisions. Nor is their attention focused only on the minimal Standard Model, because much of the book discusses nonminimal Higgs sectors, such as appear in supersymmetric and technicolour models. There are also discussions of axions, majorons and composite models. Also very useful are lengthy appendices containing detailed Feynman rules and formulae for Higgs decay rates. There is no doubt in my mind that this book should find a place in the office of any particle physicist interested in the problem of spontaneous symmetry breaking, which should include everyone working in highenergy physics.

I have only a few minor quibbles with the content of the book. It would have been good to have had a more extensive and elementary introduction to the motivation for spontaneous symmetry breaking in gauge theories and a general discussion of the Higgs mechanism. Some comparison and contrast with the theory of superconductivity could also have been appropriate, and might have made this excellent book more accessible to students and nonspecialists. Likewise, some discussion of the "naturalness" or "hierarchy" problem as a motivation for supersymmetric and technicolour models would also have been welcome.

As is perhaps inevitable in a topical book such as this, it has to some extent already been overtaken by events. Much of the discussion of a possible light Higgs boson has been rendered moot by the success of LEP at CERN, where just the Aleph experiment alone has already excluded any Standard Model Higgs boson weighing up to $41 \mathrm{GeV}$, and the combined data of all the four LEP experiments probably exclude the entire mass range up to $49 \mathrm{GeV}$. LEP has also given many limits on nonminimal Higgs bosons. By comparison, in their figure 1.1 , the authors predicted that LEP and the Stanford Linear Collider should be able to reach a mass of $30 \mathrm{GeV}$ only in 1993. Unfortunately, the SLC has been unable to muster sufficient luminosity to have any significant impact on the search for the Higgs boson.

These minor defects could easily be corrected in a second edition and do not detract from the great worth of this book, which will be an invaluable handbook until experiments find the Higgs boson or whatever replaces it, and probably long afterwards as well. This book will surely serve its avowed purpose of guiding the Higgs hunter towards her or his prey, and also interpreting whatever game she or he succeeds in bagging.

John Ellis is in the Theory Division, CERN, CH 1211 Geneva 23, Switzerland.

\section{Lucky dip}

\section{Stuart Sutherland}

Images and Understanding. Edited by Horace Barlow, Colin Blakemore and Miranda Weston-Smith. Cambridge University Press: 1990. Pp. 401. Hbk £40, $\$ 69.95$, pbk $£ 15, \$ 24.95$.

IN 1986 the Rank Prize Funds financed a conference on the use of images. Because I forgot to go, I can comment freely and without bias on the proceedings, now published somewhat tardily by Cambridge University Press under the title Images and Understanding.

The conference was nothing if not interdisciplinary for there were papers by psychologists, neurophysiologists, philosophers, computer scientists, and for good measure a writer, a theatre producer and a choreographer. Despite all the evidence refuting the belief, today even scientists seem to think that if enough people are gathered together, something good is bound to emerge. In this case what emerges is less a treasure trove than a lucky dip, which contains few pearls but has many trinkets whose glitter may amuse for a while.

Perhaps the most original contribution is that of John Willats who combines a practical knowledge of the visual arts with a formal training in engineering and a somewhat less formal one in psychology. $\mathrm{He}$ notes that paintings can attempt to represent surfaces either as seen from the observer's viewpoint or as they really are in 3-D space. Perspective is the prime example of the former category. An example of the latter is orthographic projection, in which selected surfaces are shown in their true physical shape, giving object-centred descriptions of at least part of the scene. Thus in P. Bonnard's picture, Nude in a Bath $T u b$, the top and one end of the bath are depicted in their true shapes, rather than in the shapes they would project to the retina. Willats also categorizes pictures and drawings by their denotational system, that is the rules or conventions for interpreting the marks in the picture. In a denotational system that is viewer-centred the brightness and colour of the marks reproduce an image on the retina that approximates as closely 